\title{
Combined rpoB duplex PCR and hsp65 PCR restriction fragment length polymorphism with capillary electrophoresis as an effective algorithm for identification of Mycobacterial species from clinical isolates
}

Chen-Cheng Huang ${ }^{1,9}$, Jiann-Hwa Chen², Shiau-Ting Hư ${ }^{3}$, Chien-Shun Chiou ${ }^{4}$, Wei-Chang Huang ${ }^{5}$, Jeng-Yuan Hsu ${ }^{5}$, Jang-Jih Lu ${ }^{6,9}$ and Gwan-Han Shen ${ }^{5,7,8^{*}}$

\begin{abstract}
Background: Mycobacteria can be quickly and simply identified by PCR restriction-enzyme analysis (PRA), but misidentification can occur because of similarities in band sizes that are critical for discriminating among species. Capillary electrophoresis can provide computer-aided band discrimination. The aim of this research was to develop an algorithm for identifying mycobacteria by combined rpoB duplex PRA (DPRA) and hsp65 PRA with capillary electrophoresis.

Results: Three hundred and seventy-six acid-fast bacillus smear-positive BACTEC cultures, including 200 Mycobacterium tuberculosis complexes (MTC) and 176 non-tuberculous mycobacteria (NTM) were analyzed. With combined hsp65 and rpoB DPRA, the accuracy rate was 100\% (200 isolates) for the MTC and $91.4 \%$ (161 isolates) for the NTM. Among the discordant results (8.6\%) for the NTM, one isolate of Mycobacterial species and an isolate of $M$. flavescens were found as new sub-types in hsp65 PRA.

Conclusions: This effective and novel identification algorithm using combined rpoB DPRA and hsp65 PRA with capillary electrophoresis can rapidly identify mycobacteria and find new sub-types in hsp65 PRA. In addition, it is complementary to $16 \mathrm{~S}$ rDNA sequencing.
\end{abstract}

Keywords: rpoB duplex polymerase chain reaction, hsp65 restriction fragment length polymorphism analysis, Capillary electrophoresis

\section{Background}

Detection and identification of mycobacteria in clinical specimens is a key issue in the therapy of pulmonary diseases because misidentification can lead to inappropriate treatment. Traditionally, mycobacterial species are identified based on their growth rate, presence or absence of pigmentation, and using biochemical assays of the isolates recovered from specimens. The biochemical

\footnotetext{
* Correspondence: shengwanhan@gmail.com

${ }^{5}$ Division of Respiratory and Critical Care Medicine, Department of Internal Medicine, Taichung Veterans General Hospital, Taichung, Taiwan

${ }^{7}$ Department of Respiratory Therapy, College of Health Care, China Medical University, Taiwan

Full list of author information is available at the end of the article
}

assays are time-consuming and labor-intensive, usually taking 1 to 2 months to complete, and assays for nontuberculous mycobacteria (NTM) species can have poor reproducibility and provide ambiguous results $[1,2]$.

By contrast, molecular identification, notably PCRrestriction enzyme analysis (PRA), is rapid and simple. The hsp65 PRA method, developed by Telenti et al. in 1993, is a popular DNA-based method for mycobacteria identification [3]. Using hsp65 PRA, Wong et al. [4] reported $100 \%$ sensitivity and specificity in identifying Mycobacterium tuberculosis complexes but only $74.5 \%$ sensitivity in identifying NTM species. This misidentification may occur because of similarities in band sizes that 
are critical for species discrimination [3]. An additional contributing factor is a lack of knowledge of all existing PRA profiles, especially among species that are very heterogeneous, such as $M$. gordonae, $M$. scrofulaceum, and $M$. terrae complexes. Recently, capillary electrophoresis (CE) with computer analysis [5-9] has provided more precise band discrimination than analysis by the naked eye.

Previously, we developed an algorithm for mycobacterial species identification from acid-fast bacillus (AFB) smear-positive BACTEC tubes by combining the rpoB duplex PRA (DPRA) [10] and the key phenotypic characters of mycobacteria recovered from the tubes [11]. Using rpoB DPRA, we differentiated Mycobacterium tuberculosis complexes (MTC) from NTM with 235 base pair (bp) and $136 \mathrm{bp}$ PCR amplicons in AFB smearpositive BACTEC cultures. The 136 bp rpoB duplex PCR amplicon was further digested with MspI and HaeIII (rpoB DPRA) to divide the NTM species into eight easily distinguishable groups $(\mathrm{A}-\mathrm{H})$ as described by Kim et al. [10]. Using two phenotypic characters (growth rate and photoreactivity on pigment production) and two simple biochemical assays (nitrate reduction test and Tween 80 hydrolysis test) [11], the mycobacterial species were identified. However, the sub-culture and biochemical tests for this algorithm took three weeks.

In the present study, we developed a rapid and effective algorithm for identification of mycobacteria by combined rpoB DPRA and hsp65 PRA with CE.

\section{Results}

\section{Mycobacteria identification}

There were 376 AFB smear-positive BACTEC culture tubes (positive BACTEC cultures), including $200 \mathrm{MTC}$ and 176 NTM-containing BACTEC cultures. A further
20 bacteria were MGIT positive but AFB culture smear negative, and these were classed as contaminated and excluded from subsequent evaluation.

By rpoB duplex PCR, all of the 200 MTC-containing BACTEC cultures and the 176 NTM-containing BACTEC cultures showed 235-bp and 136-bp PCR amplicons specific for MTC and NTM, respectively. The species were identified according to the flow chart shown in Figure 1.

\section{Concordant results from rpoB DPRA and hsp65 PRA}

Combining rpoB DPRA and hsp65 PRA with computeraided CE gave an accuracy rate of $100 \%$ (200/200) for MTC and 91.4\% (161/176) for NTM (Table 1).

\section{Discordant results from rpoB DPRA and $h s p 65$ PRA}

There were 15 isolates (8.6\%) of NTM with discordant results with rpoB DPRA and hsp65 PRA (Table 2). The two isolates, Mycobacterial species (A group) and $M$. flavescens (A group) identified by $16 \mathrm{~S}$ rDNA sequencing represented new patterns not available in the $h s p 65$ PRA databases and might be new sub-types in hsp65 PRA. For Mycobacterial species, 16 S rDNA sequencing did not confirm the identity of the isolate but conventional biochemical identification showed it was M. mucogenicum.

\section{Development of a species identification algorithm}

The results in Tables 1 and 2 and the mycobacterial identification flow chart (Figure 1) were used to develop a species identification algorithm by combining rpoB duplex PCR [10] and hsp65 PRA [3] using the most common 74 patterns of 40 species in Table 3 . In this algorithm (Table 3), we added $M$. gordonae types 3 and 4 to the

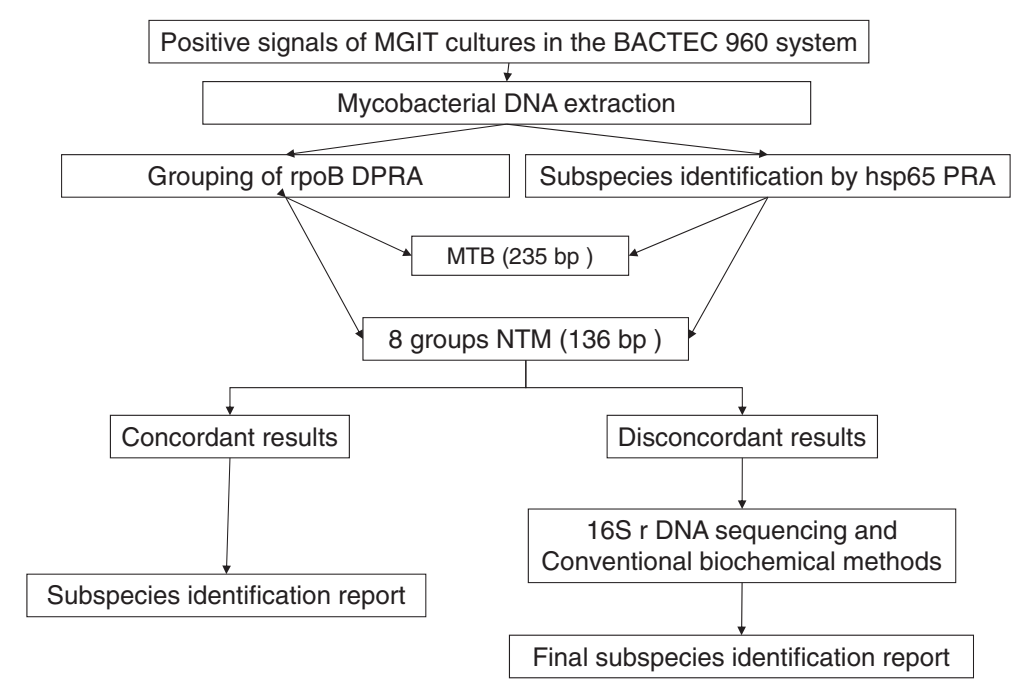

Figure 1 An flow chart of the identification of Mcobacterial species from clinical specimens by combined rpoB duplex PCR(DCR) and hsp65 PCR-Restriction Fragment Length Polymorpgism analysis(PRA). 


\begin{tabular}{|c|c|c|c|c|c|}
\hline rpoB RFLP pattern & hsp65 RFLP pattern & $\begin{array}{l}16 \text { S rDNA sequence } \\
\text { identification }\end{array}$ & $\begin{array}{c}\text { Conventional biochemical } \\
\text { identification }\end{array}$ & $\begin{array}{c}\text { BACTEC culture } \\
\text { number }\end{array}$ & $\begin{array}{c}\text { Concordance } \\
\text { rate }\end{array}$ \\
\hline \multirow[t]{2}{*}{$\mathbf{T}$} & M. tuberculosis type 1 & M. tuberculosis & M. tuberculosis & 200 & $100 \%(200 / 200)$ \\
\hline & NTM & NTM & NTM & 161 & $91.4 \%(161 / 176)$ \\
\hline A & M. abscessus type1 & M. abscessus & M. abscessus & 29 & \\
\hline A & M. abscessus type 2 & M. abscessus & M. abscessus & 41 & \\
\hline A & M. fortuitum type 1 & M. fortuitum & M. fortuitum & 33 & \\
\hline A & M. fortuitum type 2 & M. fortuitum & M. fortuitum & 2 & \\
\hline A & M. peregrinum type 1 & M. peregrinum & M. fortuitum* & 5 & \\
\hline A & M. peregrinum type 2 & M. peregrinum & M. fortuitum* & 8 & \\
\hline A & M. peregrinum type 3 & M. peregrinum & M. fortuitum* & 1 & \\
\hline A & M. chelonae type 1 & M. chelonae & M. chelonae & 1 & \\
\hline A & M. mucogenicum type 1 & M. mucogenicum & M. mucogenicum & 2 & \\
\hline A & M. smegmatis type 1 & M. smegmatis & M. smegmatis & 2 & \\
\hline B & M. avium subsp. avium type 2 & M. avium subsp. avium & M. avium complex** & 2 & \\
\hline D & M. kansasii type 1 & M. kansasii & M. kansasii & 6 & \\
\hline D & M. kansasii type 2 & M. kansasii & M. kansasii & 1 & \\
\hline D & M. kansasii type 6 & M. kansasii & M. kansasii & 1 & \\
\hline D & M. triviale type 1 & M. triviale & M. triviale & 1 & \\
\hline $\mathbf{F}$ & M. malmoense type 1 & M. malmoense & M. malmoense & 2 & \\
\hline$F$ & M. szulgai type 1 & M. szulgai & M. szulgai & 1 & \\
\hline$F$ & M. interjectum type 1 & M. interjectum & M. interjectum & 1 & \\
\hline G & M. intracellulare type 1 & M. intracellulare & M. avium complex** & 14 & \\
\hline G & M. gordonae type 1 & M. gordonae & M. gordonae & 6 & \\
\hline G & M. gordonae type 2 & M. gordonae & M. gordonae & 1 & \\
\hline G & M. gordonae type 5 & M. gordonae & M. gordonae & 1 & \\
\hline Total & & & & 361 & \\
\hline
\end{tabular}

* M. peregrinum was identified as $M$. fortuitum by a conventional biochemical method.

${ }^{*} M$. avium subsp. avium and M. intracellulare were identified as M. avium complex by a conventional biochemical method.

F group. The accuracy rate for NTM by combining the two methods could reach $96.6 \%(170 / 176)$.

\section{Discussion}

Mycobacterial species identification is generally achieved by standard culture and biochemical methods [12]. Biochemical methods are labor-intensive and time-consuming, and not all are reproducible $[2,13]$. Recently, PRA and DPRA have been developed for molecular identification of mycobacterial species using different regions of hsp65, $16 \mathrm{~S}$ rDNA, $16 \mathrm{~S}-23 \mathrm{~S}$ rDNA spacer, dnaJ, and $r p o \mathrm{~B}$ as an amplification target [3,14-17]. The most common method is hsp65 PRA, and 74 patterns for 40 species are available in the PRASITE database (http://app. chuv.ch/prasite/ index.html).

Previous studies $[18,19]$ have reported that hsp65 PRA is faster and more accurate for species identification than conventional (phenotypic or biochemical) testing. This is because more incorrect and ambiguous results are obtained with conventional methods. The results in our study (Tables 1 and 2) also support this finding. Incorrect and ambiguous results are caused by phenotypic homogeneity among different species and phenotypic variability within species [18]. With by hsp65 PRA, some sub-species, such as M. kansasii, can be identified and rapid-growing mycobacterium can be divided into M. abscessus and $M$. chelonae, M. fortuitum and M. smegmatis [20], whereas these identifications are difficult with conventional methods [21]. As found in our study (Tables 1 and 2), M. peregrinum was identified as M. fortuitum and M. avium subsp. avium and $M$. intracellulare were both identified as $M$. avium complex by the conventional biochemical method.

However, $h s p 65$ PRA limitations have been reported in some articles $[22,23]$. Failure to identify or incorrect 


\begin{tabular}{|c|c|c|c|c|}
\hline No & rpoB RFLP pattern & hsp65 RFLP pattern & $16 \mathrm{~S}$ rDNA sequence & Conventional biochemical identification \\
\hline \multirow[t]{2}{*}{1} & A & BstEIl : 242.8* $214.0,0$ & M. flavescens & M. flavescens \\
\hline & & Haelll: 130.9, 140, 90.4, 49.7, 41.5, 37.1 & & \\
\hline \multirow[t]{2}{*}{2} & A & BstEll :456.3, 0, 0 & Mycobacterial species & M. mucogenicum \\
\hline & & Haelll:192.6, 90.4, 82.0 & & \\
\hline 3 & D & M. scrofulaceum type 1 & M. scrofulaceum & M. scrofulaceum \\
\hline 4 & G & M. simiae type 5 & M. simiae & M. simiae \\
\hline 5 & G & M. simiae type 5 & M. simiae & M. simiae \\
\hline 6 & $\mathbf{F}$ & M. intracellulare type 3 & M. intracellulare & M. avium complex ${ }^{* *}$ \\
\hline 7 & $\mathbf{F}$ & M. gordonae type 3 & M. gordonae & M. gordonae \\
\hline 8 & $\mathbf{F}$ & M. gordonae type 3 & M. gordonae & M. gordonae \\
\hline 9 & $\mathbf{F}$ & M. gordonae type 3 & M. gordonae & M. gordonae \\
\hline 10 & $\mathbf{F}$ & M. gordonae type 3 & M. gordonae & M. gordonae \\
\hline 11 & $F$ & M. gordonae type 3 & M. gordonae & M. gordonae \\
\hline 12 & $\mathbf{F}$ & M. gordonae type 3 & M. gordonae & M. gordonae \\
\hline 13 & $\mathrm{~F}$ & M. gordonae type 3 & M. gordonae & M. gordonae \\
\hline 14 & $\mathbf{F}$ & M. gordonae type 4 & M. gordonae & M. gordonae \\
\hline 15 & $\mathrm{~F}$ & M. gordonae type 4 & M. gordonae & M. gordonae \\
\hline
\end{tabular}

** M. avium subsp. avium and M. intracellulare were identified as M. avium complex by a conventional biochemical method.

identification of the species may occur because of similarities in band sizes critical for discriminating species, including difficult to distinguish $M$. tuberculosis complex (M. tuberculosis and M. bovis) [22], and closely related sub-species such as M. avium or M. gordonae, because of sequence heterogeneity [22]. In addition, technical problems can also cause misinterpretation or incorrect identification [23]. Patterns in PRA profiles are complex and difficult to interpret with the naked eye, especially when more detailed sub-types are included [21].

This study combined rpoB DPRA and hsp65 PRA to test both reference strains and clinical respiratory isolates. The mycobacterial identification flow chart (Figure 1) can identify species to the sub-species level, and final species identification can be obtained instantly with concordant results from the two PRA. M. gordonae has a highly variable gene sequence with 10 sub-types in hsp65 PRA, and there are two groups (G and F) in rpoB DPRA. Most M. gordonae is in the $\mathrm{G}$ group, but $M$. gordonae types 3 and 4 by $h s p 65$ PRA are in the F group (Tables 1 and 2).

In addition, there were different rpoB DPRA results (Table 2) for $M$. simaie type 5 (G group but not E group), M. scrofulaceum type 1 (D group but not $\mathrm{H}$ group), and $M$. intracellulare type 3 (F group but not $\mathrm{G}$ group). The identities of all of these isolates were finally confirmed by $16 \mathrm{~S}$ rDNA sequencing. Variable numbers of restriction sites for HaeIII in these species may derive from genetic sequence mutation. However, these species are included in the species identification algorithm even though they are uncommon isolates.

Using the mycobacteria identification flow chart (Figure 1) and algorithm (Table 3), M. avium-intracellulare complex (MAC) can be easily divided into M. avium spp. avium and M. intracellulare by both rpoB DPRA and hsp 65 PRA. By contrast, this was not possible with the conventional method. Using the results in Table 3, some NTM species with identical or similar $h s p 65$ PRA can be clearly grouped by rpoB DPRA (Table 4). Ambiguous results from $h s p 65$ PRA alone are easier to interpret with combined rpoB DPRA and hsp65 PRA. However, M. intermedium type 1 and $M$. intracellulare type 3 with identical hsp65 PRA and rроB DPRA (G group) could not be differentiated further by this species identification algorithm and required $16 \mathrm{~S}$ rDNA sequencing for confirmation.

Although $16 \mathrm{~S}$ rDNA sequencing is the standard method for mycobacterium species identification, it cannot differentiate some closely related rapid-growing mycobacterium species [24] or slow-growing M. kansasii and M. gastri that had identical $16 \mathrm{~S}$ rDNA sequences, but these can be differentiated by $h s p 65$ PRA and rpoB DPRA. There are some reports [6,25] of conflicting results from different methods for mycobacterial species identification, probably caused by a failure of one method to identify all test strains correctly. Combining methods for mycobacterial species identification can improve the accuracy rate, avoid ambiguous results, and save time. 
Table 3 A species identification algorithm by combining rpoB duplex PCR and hsp65 PCR-restriction fragment length polymorphism analysis

\begin{tabular}{|c|c|c|c|c|c|}
\hline \multirow[b]{2}{*}{ Pattern } & \multicolumn{2}{|c|}{ rpoB DPCR-RFLP } & \multicolumn{2}{|l|}{ hsp65 RFLP } & \multirow[t]{2}{*}{ Final species identification } \\
\hline & Msp I & Haelll & BstEll & Hae III & \\
\hline \multirow[t]{29}{*}{$A$} & 136 & 136 & $440 / 0 / 0$ & $160 / 90 / 60 / 0$ & M. vaccae type 1 \\
\hline & & & $440 / 0 / 0$ & $160 / 85 / 55 / 0$ & M. flavescens type 3 \\
\hline & & & $440 / 0 / 0$ & $140 / 55 / 50 / 0$ & M. flavescens type 1 \\
\hline & & & $440 / 0 / 0$ & $130 / 115 / 70 / 60$ & M. aurum type 2 \\
\hline & & & $320 / 130 / 0$ & $200 / 70 / 60 / 55$ & M. immunogenum type 2 \\
\hline & & & $320 / 130 / 0$ & $200 / 60 / 55 / 50$ & M. chelonae type 1 \\
\hline & & & $320 / 130 / 0$ & $145 / 70 / 60 / 55$ & M. immunogenum type 1 \\
\hline & & & $320 / 130 / 0$ & $140 / 65 / 60 / 0$ & M. mucogenicum type 1 \\
\hline & & & $320 / 115 / 0$ & $185 / 145 / 0 / 0$ & M. fallax type 1 \\
\hline & & & $320 / 115 / 0$ & $170 / 140 / 0 / 0$ & M. neoaurum type 1 \\
\hline & & & $320 / 115 / 0$ & $145 / 65 / 60 / 0$ & M. mucogenicum type 2 \\
\hline & & & $320 / 115 / 0$ & $140 / 90 / 60 / 0$ & M. mucogenicum type 3 \\
\hline & & & $235 / 210 / 0$ & $200 / 70 / 60 / 50$ & M. abscessus type 2 \\
\hline & & & $235 / 210 / 0$ & $180 / 135 / 70 / 50$ & M. thermoresistibile type 1 \\
\hline & & & $235 / 210 / 0$ & $145 / 140 / 100 / 50$ & M. peregrinum type 1 \\
\hline & & & $235 / 210 / 0$ & $145 / 70 / 60 / 55$ & M. abscessus type 1 \\
\hline & & & $235 / 210 / 0$ & $140 / 125 / 100 / 50$ & M. peregrinum type 2 \\
\hline & & & $235 / 210 / 0$ & $140 / 125 / 60 / 50$ & M. senegalense type 3 \\
\hline & & & $235 / 210 / 0$ & $140 / 80 / 60 / 50$ & M. phlei type 1 \\
\hline & & & $235 / 210 / 0$ & $130 / 80 / 60 / 0$ & M. celatum type 1 \\
\hline & & & $235 / 130 / 85$ & $175 / 80 / 0 / 0$ & M. aurum type 1 \\
\hline & & & $235 / 130 / 85$ & $145 / 140 / 100 / 60$ & M. peregrinum type 3 \\
\hline & & & $235 / 130 / 85$ & $145 / 125 / 60 / 0$ & M. smegmatis type 1 \\
\hline & & & $235 / 130 / 85$ & $140 / 125 / 60 / 50$ & M. senegalense type 2 \\
\hline & & & $235 / 120 / 85$ & $180 / 140 / 50 / 0$ & M. senegalense type 4 \\
\hline & & & $235 / 120 / 85$ & $145 / 120 / 60 / 55$ & M. fortuitum type 1 \\
\hline & & & $235 / 120 / 85$ & $140 / 125 / 60 / 50$ & M. senegalense type 1 \\
\hline & & & $235 / 120 / 85$ & $140 / 120 / 60 / 55$ & M. fortuitum type 2 \\
\hline & & & $235 / 120 / 85$ & $135 / 90 / 85 / 0$ & M. fortuitum type 3 \\
\hline \multirow[t]{5}{*}{ B } & 136 & 108,28 & $320 / 115 / 0$ & $140 / 90 / 60 / 0$ & M. chitae type 1 \\
\hline & & & $235 / 210 / 0$ & $145 / 130 / 0 / 0$ & M. avium subsp. avium type 3 \\
\hline & & & $235 / 210 / 0$ & $130 / 105 / 60 / 0$ & M. avium subsp. avium type 2 \\
\hline & & & $235 / 210 / 0$ & $130 / 105 / 0 / 0$ & M. avium subsp. avium type 1 \\
\hline & & & $235 / 210 / 0$ & $130 / 105 / 0 / 0$ & M. avium subsp. paratuberculosis type 1 \\
\hline C & 136 & 76,60 & $235 / 120 / 85$ & $160 / 105 / 60 / 0$ & M. xenopi type 1 \\
\hline \multirow[t]{7}{*}{ D } & 75,61 or & 136 & $440 / 0 / 0$ & $170 / 130 / 0 / 0$ & M. triviale type 1 \\
\hline & $75,57,4$ & & $320 / 115 / 0$ & 130 / 95 / 75 / 60 & M. kansasii type 5 \\
\hline & & & $235 / 210 / 0$ & $190 / 105 / 80 / 0$ & M. ulcerans type 2 \\
\hline & & & $(235 / 210 / 0$ & $145 / 130 / 95 / 0$ & M. scrofulaceum type $1^{*}$ ) \\
\hline & & & $235 / 210 / 0$ & $145 / 105 / 80 / 45 / 20$ & M. marinum type 1 \\
\hline & & & $235 / 210 / 0$ & $145 / 105 / 80 / 0$ & M. ulcerans type 1 \\
\hline & & & $235 / 210 / 0$ & $130 / 105 / 80 / 0$ & M. kansasii type 1 \\
\hline
\end{tabular}


Table 3 A species identification algorithm by combining rpoB duplex PCR and hsp65 PCR-restriction fragment length polymorphism analysis (Continued)

\begin{tabular}{|c|c|c|c|c|c|}
\hline & & & $235 / 130 / 85$ & $140 / 105 / 70 / 0$ & M. shimodei type 1 \\
\hline & & & $235 / 120 / 85$ & $130 / 115 / 75 / 60$ & M. kansasii type 4 \\
\hline & & & $235 / 130 / 85$ & $130 / 105 / 70 / 0$ & M. kansasii type 6 \\
\hline & & & $235 / 130 / 85$ & $130 / 105 / 0 / 0$ & M. kansasii type 2 \\
\hline & & & $235 / 130 / 85$ & $130 / 95 / 70 / 0$ & M. kansasii type 3 \\
\hline \multirow[t]{15}{*}{ E } & 75,61 or & 108,28 & $440 / 0 / 0$ & $145 / 130 / 0 / 0$ & M. simiae type 5 \\
\hline & $75,57,4$ & & $320 / 115 / 0$ & $185 / 140 / 0 / 0$ & M. terrae type 2 \\
\hline & & & $320 / 115 / 0$ & $180 / 130 / 0 / 0$ & M. terrae type 1 \\
\hline & & & $320 / 115 / 0$ & $145 / 130 / 0 / 0$ & M. simiae type 4 \\
\hline & & & $320 / 115 / 0$ & $140 / 90 / 60 / 0$ & M. nonchromogenicum type 2 \\
\hline & & & $320 / 115 / 0$ & $140 / 60 / 50 / 0$ & M. terrae type 3 \\
\hline & & & $320 / 115 / 0$ & $125 / 105 / 0 / 0$ & M. genavense type 1 \\
\hline & & & $235 / 210 / 0$ & $185 / 130 / 0 / 0$ & M. simiae type 1 \\
\hline & & & $235 / 210 / 0$ & $185 / 130 / 0 / 0$ & M. genavense type 2 \\
\hline & & & $235 / 210 / 0$ & $155 / 140 / 0 / 0$ & M. simiae type 2 \\
\hline & & & $235 / 210 / 0$ & $145 / 130 / 0 / 0$ & M. simiae type 6 \\
\hline & & & $235 / 210 / 0$ & $140 / 115 / 70 / 0$ & M. terrae type 4 \\
\hline & & & $235 / 130 / 85$ & $145 / 130 / 0 / 0$ & M. simiae type 3 \\
\hline & & & $235 / 130 / 85$ & $130 / 105 / 70 / 0$ & M. gastri type 1 \\
\hline & & & $235 / 120 / 85$ & $145 / 60 / 55 / 0$ & M. nonchromogenicumtype 1 \\
\hline \multirow[t]{9}{*}{$\mathbf{F}$} & 75,61 or & 76,60 & $440 / 0 / 0$ & $130 / 105 / 70 / 0$ & M. szulgai type 1 \\
\hline & $75,57,4$ & & $(320 / 115 / 0$ & $130 / 115 / 60 / 0$ & M. gordonae type $4^{*}$ ) \\
\hline & & & $240 / 210 / 0$ & 130/110/0 & M. interjectum \\
\hline & & & $(235 / 210 / 0$ & $145 / 130 / 0 / 0$ & M. intracellulare type $3^{*}$ ) \\
\hline & & & $235 / 210 / 0$ & $115 / 105 / 0 / 0$ & M. asiaticum type 1 \\
\hline & & & $235 / 130 / 85$ & $130 / 105 / 80 / 0$ & M. celatum type 2 \\
\hline & & & $235 / 120 / 100$ & $145 / 105 / 80 / 0$ & M. malmoense type 1 \\
\hline & & & $235 / 210 / 0$ & $145 / 105 / 80 / 0$ & M. malmoense type 2 \\
\hline & & & $(235 / 120 / 100$ & $130 / 115 / 0 / 0$ & M. gordonae type $3^{*}$ ) \\
\hline \multirow[t]{15}{*}{ G } & 75,61 or & $76,32,28$ & $(440 / 0 / 0$ & $145 / 130 / 0 / 0$ & M. simiae type $5^{*}$ ) \\
\hline & $75,57,4$ & & $320 / 115 / 0$ & $130 / 110 / 70 / 60$ & M. gordonae type 8 \\
\hline & & & $320 / 115 / 0$ & $130 / 115 / 60 / 0$ & M. gordonae type 4 \\
\hline & & & $235 / 210 / 0$ & $145 / 130 / 0 / 0$ & M. intermedium type 1 \\
\hline & & & $235 / 210 / 0$ & $145 / 130 / 0 / 0$ & M. intracellulare type 3 \\
\hline & & & $235 / 210 / 0$ & $140 / 105 / 80 / 0$ & M. intracellulare type 2 \\
\hline & & & $235 / 210 / 0$ & $130 / 115 / 0 / 0$ & M. gordonae type 5 \\
\hline & & & $235 / 210 / 0$ & $120 / 115 / 110 / 0$ & M. intracellulare type 4 \\
\hline & & & $235 / 130 / 85$ & $140 / 120 / 95 / 0$ & M. gordonae type 6 \\
\hline & & & $235 / 120 / 100$ & $160 / 115 / 60 / 0$ & M. gordonae type 9 \\
\hline & & & $235 / 120 / 100$ & $155 / 110 / 0 / 0$ & M. gordonae type 7 \\
\hline & & & $235 / 120 / 100$ & $145 / 130 / 60 / 0$ & M. intracellulare type 1 \\
\hline & & & $235 / 120 / 100$ & $130 / 115 / 0 / 0$ & M. gordonae type 3 \\
\hline & & & $235 / 120 / 100$ & $130 / 110 / 95 / 0$ & M. gordonae type 10 \\
\hline & & & $235 / 120 / 85$ & $160 / 115 / 60 / 0$ & M. gordonae type 1 \\
\hline
\end{tabular}


Table 3 A species identification algorithm by combining rpoB duplex PCR and hsp65 PCR-restriction fragment length polymorphism analysis (Continued)

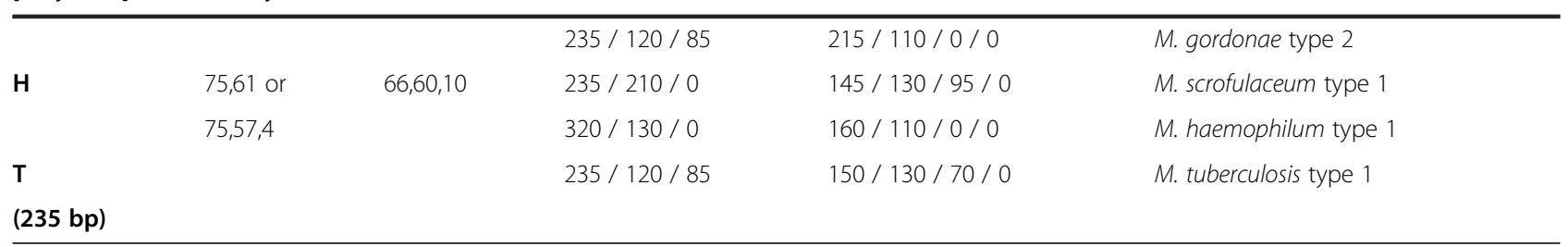

$\left.{ }^{*}\right)$ species from Table 2 were included in this algorithm.

Many CE-based studies [5-9] in PCR-RFLP analysis have investigated improving band size discrimination. In one study by Chang et al. [7], high-resolution CE gave more precise estimates of DNA fragment sizes than analysis by the naked eye, and CE could detect low molecular weight fragments (down to $12 \mathrm{bp}$ ). In our study, restriction fragments $<50 \mathrm{bp}$ were not selected for analysis to avoid confusion with primer and primer dimer bands. We found that more hsp65 fragment differences than rpoB fragment (data not shown) may explain the size differences with highly variable sequence for species identification but difficult interpretation in hsp65 PRA.

Some sub-types of NTM species are relevant to clinical management, such as the M. kansasii and MAC. $M$. kansasii type 1 is the most common type associated with human disease [26-28] because of its high pathogenicity. However, M. kansasii types 3-7 are most often isolated

Table 4 Species with identical or similar hsp65 PRA but different groups in rpoB DPRA

\begin{tabular}{llll}
\hline rpoB & Species (type) & \multicolumn{2}{l}{ hsp65 RFLP } \\
\cline { 3 - 4 } & & BstEII & Hae III \\
\hline A & M. mucogenicum type 3 & $320 / 115 / 0$ & $140 / 90 / 60$ / \\
B & M. chitae type 1 & $320 / 115 / 0$ & $140 / 90 / 60 / 0$ \\
A & M. mucogenicum type 2 & $320 / 115 / 0$ & $145 / 65 / 60 / 0$ \\
E & M. terrae type 3 & $320 / 115 / 0$ & $140 / 60 / 50 / 0$ \\
A & M. fallax type 1 & $320 / 115 / 0$ & $185 / 145 / 0 / 0$ \\
E & M. terrae type 2 & $320 / 115 / 0$ & $185 / 140 / 0 / 0$ \\
A & M. peregrinum type 2 & $235 / 210 / 0$ & $140 / 125 / 100 / 50$ \\
H & M. scrofulaceum type 1 & $235 / 210 / 0$ & $145 / 130 / 95 / 0$ \\
D & M. kansasii type 6 & $235 / 130 / 85$ & $130 / 105 / 70 / 0$ \\
E & M. gastri type 1 & $235 / 130 / 85$ & $130 / 105 / 70 / 0$ \\
F & M. celatum type 2 & $235 / 130 / 85$ & $130 / 105 / 80 / 0$ \\
D & M. kansasii type 1 & $235 / 210 / 0$ & $130 / 105 / 80 / 0$ \\
F & M. malmoense type 2 & $235 / 210 / 0$ & $145 / 105 / 80 / 0$ \\
E & M. simiae type 6 & $235 / 210 / 0$ & $145 / 130 / 0 / 0$ \\
G & M. intermedium type 1 & $235 / 210 / 0$ & $145 / 130 / 0 / 0$ \\
G & M. intracellulare type 3 & $235 / 210 / 0$ & $145 / 130 / 0 / 0$ \\
F & M. interjectum & $240 / 210 / 0$ & $130 / 110 / 0$ \\
G & M. gordonae type 5 & $235 / 210 / 0$ & $130 / 115 / 0 / 0$ \\
\hline & & &
\end{tabular}

from the environment and rarely from humans, and have no significant role in clinical management $[26,27]$. MAC can be divided into M. avium subsp. avium and $M$. intracellulare because drug sensitivity test and clinical outcomes are different between these two subtypes $[29,30]$.

It is important to identify NTM to the sub-type level both for epidemiologic data and for differentiating potentially pathogenic sub-types [26,27]. Combined rpoB DPRA and hsp65 PRA with capillary electrophoresis provides precise species identification and overcomes problems associated with discrimination by hsp 65 PRA band sizes. This combined method takes around 2-3 days to complete in the laboratory once clinical isolates have been received. However, the identification algorithm has some limitations. First, it could not discriminate $M$. intermedium type 1 from $M$. intracellulare type 3, and second, not every hospital laboratory will be equipped with the appropriate equipment for this method.

\section{Conclusion}

In conclusion, the novel flow chart and identification algorithm obtained by combined rpoB DPRA and $h s p 65$ PRA with capillary electrophoresis can easily differentiate MTC from NTM and identify mycobacterial species to the sub-type level, which is helpful for clinical management. The results are complementary to $16 \mathrm{~S}$ rDNA sequencing, and the effective algorithm provides rapid and accurate mycobacterial species identification.

\section{Methods}

\section{Mycobacterial isolates}

Fourteen mycobacterial reference strains including one MTC and 13 NTM strains and 376 clinical respiratory specimens, including sputum, broncho-alveolar lavage, and aspirated secretion from endotracheal tubes, were collected from January to July 2007 from Taichung Veterans General Hospital (Taichung, Taiwan). The respiratory specimens were digested by a $\mathrm{N}$-acetyl-L-cysteine- $\mathrm{NaOH}$ decontamination procedure, centrifugal concentration, and sputum dissolving agents [31]. The processed specimens or the concentrated specimens were inoculated into MGIT culture tubes and incubated in the BACTEC 960 instrument at $37^{\circ} \mathrm{C}$ until a positive signal appeared. 
Positive BACTEC cultures were smeared on glass slides and Kinyoun staining was used to screen for AFB [31]. Mycobacteria in the positive BACTEC cultures were isolated and identified by conventional methods $[12,13]$.

\section{Identification of mycobacterial isolates by conventional methods}

All 376 isolates from cultures in the AFB smear-positive BACTEC tubes were streaked on Löwenstein-Jensen (LJ) slants and incubated at $37^{\circ} \mathrm{C}$ under $5 \% \mathrm{CO}_{2}$. Colonies on the LJ slants were used for species identification by conventional culture and biochemical methods $[12,13]$. These methods included growth rates, photoreactivity for pigment production, morphology in microcolonies on $\mathrm{LJ}$ slants, and biochemical tests, including nitrate reduction, arylsulfatase, Tween 80 hydrolysis, urease, semiquantitative catalase, tolerance to $5 \% \mathrm{NaCl}$ and niacin production.

\section{Genomic DNA extraction}

Mycobacterial DNA was extracted from positive BACTEC cultures using a DTB specimen processing kit (Becton Dickinson, Franklin Lakes, NJ) according to the manufacturer's instructions [11].

\section{rpoB DPCR and rpoB DPRA}

The $\mathrm{r} p o \mathrm{~B}$ DPCR was performed using genomic DNA as template and primer pairs Tbc1 (5'-CGTACGGTCGGCG AGCTGATCCAA-3')-TbcR5 (5'-CCACCAGTCGGCGC TTGTGGGTCAA-3') and M5 (5'-GGAGCGGATGACC ACCCAGGACGTC-3')-RM3 (5'-CAGCGGGTT GTTC TGGTCCATGAAC-3') as described by Kim et al. [10]. A 235 bp DNA PCR amplicon from MTC and a 136 bp DNA PCR amplicon from NTM were specifically amplified [10], and these two amplification products were analyzed by electrophoresis on a $2 \%$ agarose gel (Seakem LE agarose, Cambrex, East Rutherford, NJ).

For rpoB DPRA, the 136-bp DNA PCR amplicon was further digested with MspI and HaeIII after DPRA, and analyzed by electrophoresis on a $3 \%$ agarose gel (NuSieve 3:1 agarose, Cambrex) or CE (eGene). The rpoB restriction fragment length polymorphism (RFLP) patterns were compared to eight groups described by Kim et al. [10]. Eight NTM reference strains (M. abscessus ATCC 19977, M. avium subsp. avium ATCC 25291, M. kansasii ATCC 12479, M. terrae ATCC 15755, M. szulgai ATCC 29716, M. intracellulare ATCC 13950, M. scrofulaceum ATCC 19981, M. xenopi ATCC 19250) from each rpoB group (A-H) were subjected to rpoB DPRA by CE (eGene).

\section{hsp65 PCR and hsp65PRA}

The hsp65 PCR was performed using genomic DNA as template and primer Tb11(5'-ACC AAC GAT GGT GTG TCC-3') and Tb12 (5'-CTT GTC GAA CCG CAT ACC CT-3') as described by Telenti et al. [3]. A 439-bp DNA hsp65 PCR amplicon was specifically amplified from the extracted DNA, and the amplification product was analyzed by electrophoresis on a $2 \%$ agarose gel (Seakem LE agarose, Cambrex).

For hsp65 PRA, the 439-bp DNA hsp65 PCR amplicon was further digested with BstEII and HaeIII after completing $h s p 65$ PCR, and analyzed by electrophoresis on a 3\% agarose gel (NuSieve 3:1 agarose, Cambrex) or by $\mathrm{CE}$ (eGene). The sizes of the restriction fragment by $h s p 65$ PRA were compared to those reported on the PRASITE database (http://app.chuv.ch/prasite/index.html). Thirteen ATCC NTM reference strains and one MTC reference strain were subjected to $h s p 65$ PRA by CE (eGene).

\section{eGene CE}

The restriction fragment sizes were estimated by the naked eye or by eGene CE according to the manufacturer's instructions using size markers (100 bp to $3 \mathrm{~kb}$ ) and alignment markers (15 bp to $3 \mathrm{~kb}$ ).

\section{Mycobacterial identification flow chart}

The mycobacterial identification flow chart is shown in Figure 1.

\section{$16 S$ rDNA sequencing}

The $16 \mathrm{~S}$ rDNA sequencing of mycobacterial DNA as the reference standard method for mycobacterial species identification was carried out using primer pair 8FPL (5’AGTTTGATCCTGGCTCAG 3') and 1492 (5'GGTT ACCTTGTTACGACT T 3') as described by Turenne et al. [32]. The species were identified by comparing the $16 \mathrm{~S}$ rDNA sequences with similar sequences from GenBank.

\section{Abbreviations}

PRA: Polymerase chain reaction restriction-enzyme analysis; DPRA: Duplex Polymerase chain Reaction restriction-enzyme Analysis; MTC: Mycobacterium Tuberculosis Complexes; NTM: Non-Tuberculous Mycobacteria; CE: Capillary Electrophoresis; AFB: Acid-Fast Bacillus; MAC: M. Avium-intracellulare Complex; LJ: Löwenstein-Jensen.

\section{Competing interest}

The authors declare that they have no competing interests.

\section{Authors' contributions}

CCH wrote the manuscript. CSC, JHC, STH participated in the study design, and analysis. GHS and WCH managed the project. JYH, JJ assisted in improving the manuscript. All authors read and approved the final manuscript.

\section{Acknowledgements}

This work was supported by grants from the Center of Disease Control (Grant No. DOH95-DC-1106) and the National Science Foundation (Grant No. NSC-982A52) of Taiwan.

\section{Author details}

'Department of internal medicine, Executive Yuan Department of health, Division of Respiratory and Critical Care Medicine, Taichung Hospital, Taichung, Taiwan. ${ }^{2}$ Institute of Molecular Biology, National Chung Hsing University, Taichung 402, Taiwan. ${ }^{3}$ Institute of Microbiology and Immunology, National Yang-Ming University, Taipei, Taiwan. ${ }^{4}$ The Central Region

Laboratory, Centers for Disease Control, Department of Health, Taichung 408, 
Taiwan. ${ }^{5}$ Division of Respiratory and Critical Care Medicine, Department of Internal Medicine, Taichung Veterans General Hospital, Taichung, Taiwan. ${ }^{6}$ Department of Laboratory Medicine, Linkou Chang-Gung Memorial Hospital, Taoyuan, Taiwan. ${ }^{7}$ Department of Respiratory Therapy, College of Health Care, China Medical University, Taiwan. ${ }^{8}$ Institute of Nursing Care, Hungeuang University, Taichung, Taiwan. ${ }^{9}$ Graduate Institute of Clinical Medical Science, China Medical University, Taichung, Taiwan.

Received: 12 January 2012 Accepted: 25 June 2012

Published: 8 July 2012

\section{References}

1. Collins $\mathrm{CH}$, Grange JM, Yates MD: Tuberculosis bacteriology: organization and practice. 2nd edition. Oxford; Boston: Butterworth-Heinemann; 1997.

2. Springer B, Stockman L, Teschner K, Roberts GD, Bottger EC: Two-laboratory collaborative study on identification of mycobacteria: molecular versus phenotypic methods. J Clin Microbiol 1996, 34:296-303.

3. Telenti A, Marchesi F, Balz M, Bally F, Bottger EC, Bodmer T: Rapid identification of mycobacteria to the species level by polymerase chain reaction and restriction enzyme analysis. J Clin Microbiol 1993, 31:175-178.

4. Wong DA, Yip PC, Tse DL, Tung WW, Cheung DT, Kam KM: Routine use of a simple low-cost genotypic assay for the identification of mycobacteria in a high throughput laboratory. Diagn Microbiol Infect Dis 2003, 47:421-426.

5. Chang PL, Hsieh WS, Chiang CL, Yen-Liberman B, Procop GW, Chang HT, Ho HT: Identification of individual DNA molecule of Mycobacterium tuberculosis by nested PCR-RFLP and capillary electrophoresis. Talanta 2008, 77:182-188.

6. Sajduda A, Martin A, Portaels F, Palomino JC: hsp65 PCR-restriction analysis (PRA) with capillary electrophoresis in comparison to three other methods for identification of Mycobacterium species. J Microbiol Methods 2010, 80:190-197.

7. Chang PL, Hsieh WS, Chiang CL, Tuohy MJ, Hall GS, Procop GW, Chang HT, Ho HT: The hsp65 gene patterns of less common Mycobacterium and Nocardia spp. by polymerase chain reaction-restriction fragment length polymorphism analysis with capillary electrophoresis. Diagn Microbiol Infect Dis 2007, 58:315-323.

8. Yokoyama E, Kishida K, Uchimura M, Ichinohe S: Comparison between agarose gel electrophoresis and capillary electrophoresis for variable numbers of tandem repeat typing of Mycobacterium tuberculosis. J Microbiol Methods 2006, 65:425-431.

9. Lindstedt BA, Vardund T, Aas L, Kapperud G: Multiple-locus variablenumber tandem-repeats analysis of Salmonella enterica subsp. enterica serovar Typhimurium using PCR multiplexing and multicolor capillary electrophoresis. J Microbiol Methods 2004, 59:163-172.

10. Kim BJ, Hong SK, Lee KH, Yun YJ, Kim EC, Park YG, Bai GH, Kook YH: Differential identification of Mycobacterium tuberculosis complex and nontuberculous mycobacteria by duplex PCR assay using the RNA polymerase gene (rpoB). J Clin Microbiol 2004, 42:1308-1312.

11. Shen GH, Hung CH, Hu ST, Wu BD, Lin CF, Chen CH, Wu KM, Chen JH: Combining polymerase chain reaction restriction enzyme analysis with phenotypic characters for mycobacteria identification in Taiwan. Int J Tuberc Lung Dis 2009, 13:472-479.

12. Witebsky FG, Kruczak-Filipov P: Identification of mycobacteria by conventional methods. Clin Lab Med 1996, 16:569-601.

13. Vossler JL: Mycobacterium tuberculosis and other non-tuberculous mycobacteria. In Text-book of diagnostic microbiology. Edited by Mahon CRMG. Philadephia, PA, USA: W B Saunders; 2000:667-707.

14. Domenech P, Menendez MC, Garcia MJ: Restriction fragment length polymorphisms of $16 \mathrm{~S}$ rRNA genes in the differentiation of fast-growing mycobacterial species. FEMS Microbiol Lett 1994, 116:19-24.

15. Lee H, Park HJ, Cho SN, Bai GH, Kim SJ: Species identification of mycobacteria by PCR-restriction fragment length polymorphism of the rpoB gene. J Clin Microbiol 2000, 38:2966-2971.

16. Roth A, Reischl U, Streubel A, Naumann L, Kroppenstedt RM, Habicht M, Fischer M, Mauch H: Novel Diagnostic Algorithm for Identification of Mycobacteria Using Genus-Specific Amplification of the 16 S-23S rRNA Gene Spacer and Restriction Endonucleases. J Clin Microbiol 2000, 38:1094-1104.

17. Takewaki S, Okuzumi K, Manabe I, Tanimura M, Miyamura K, Nakahara K, Yazaki Y, Ohkubo A, Nagai R: Nucleotide sequence comparison of the mycobacterial dnaJ gene and PCR-restriction fragment length polymorphism analysis for identification of mycobacterial species. Int J Syst Bacteriol 1994, 44:159-166.

18. Chimara E, Ferrazoli L, Ueky SY, Martins MC, Durham AM, Arbeit RD, Leao SC: Reliable identification of mycobacterial species by PCR-restriction enzyme analysis (PRA)-hsp65 in a reference laboratory and elaboration of a sequence-based extended algorithm of PRA-hsp65 patterns. BMC Microbiol 2008, 8:48.

19. Kim BJ, Park JH, Lee SA, Kim H, Cha CY, Kook YH, Kim EC, Joo SI, Lee JS, Yim JJ: Differentiation of mycobacteria in sputa by duplex polymerase chain reaction for mycobacterial hsp65 gene. Diagn Microbiol Infect Dis 2008, 62:193-198.

20. Brown-Elliott BA, Wallace RJ Jr: Clinical and taxonomic status of pathogenic nonpigmented or late-pigmenting rapidly growing mycobacteria. Clin Microbiol Rev 2002, 15:716-746.

21. Kim HJ, Mun HS, Kim H, Oh EJ, Ha Y, Bai GH, Park YG, Cha CY, Kook YH, Kim BJ: Differentiation of Mycobacterial species by hsp65 duplex PCR followed by duplex-PCR-based restriction analysis and direct sequencing. J Clin Microbiol 2006, 44:3855-3862.

22. McNabb A, Eisler D, Adie K, Amos M, Rodrigues M, Stephens G, Black WA Isaac-Renton J: Assessment of partial sequencing of the 65-kilodalton heat shock protein gene (hsp65) for routine identification of Mycobacterium species isolated from clinical sources. J Clin Microbiol 2004, 42:3000-3011.

23. Leao SC, Bernardelli A, Cataldi A, Zumarraga M, Robledo J, Realpe T, Mejia Gl, da Silva Telles MA, Chimara E, Velazco M, et al: Multicenter evaluation of mycobacteria identification by PCR restriction enzyme analysis in laboratories from Latin America and the Caribbean. J Microbiol Methods 2005, 61:193-199.

24. Ringuet H, Akoua-Koffi C, Honore S, Varnerot A, Vincent V, Berche P, Gaillard $J \mathrm{~L}$, Pierre-Audigier C: hsp65 sequencing for identification of rapidly growing mycobacteria. J Clin Microbiol 1999, 37:852-857.

25. Häfner B, Haag H, Geiss H-K, Nolte O: Different molecular methods for the identification of rarely isolated non-tuberculous mycobacteria and description of new hsp65 restriction fragment length polymorphism patterns. Mol Cell Probes 2004, 18:59-65.

26. da Silva Telles MA, Chimara E, Ferrazoli L, Riley LW: Mycobacterium kansasii: antibiotic susceptibility and PCR-restriction analysis of clinical isolates. J Med Microbiol 2005, 54:975-979.

27. Taillard C, Greub G, Weber R, Pfyffer GE, Bodmer T, Zimmerli S, Frei R, Bassetti S, Rohner P, Piffaretti JC, et al: Clinical implications of Mycobacterium kansasii species heterogeneity: Swiss National Survey. J Clin Microbiol 2003, 41:1240-1244.

28. Zhang $Y$, Mann LB, Wilson RW, Brown-Elliott BA, Vincent $V$, linuma $Y$, Wallace RJ Jr: Molecular analysis of Mycobacterium kansasii isolates from the United States. J Clin Microbiol 2004, 42:119-125.

29. Maekura R, Okuda Y, Hirotani A, Kitada S, Hiraga T, Yoshimura K, Yano I, Kobayashi K, Ito M: Clinical and prognostic importance of serotyping Mycobacterium avium-Mycobacterium intracellulare complex isolates in human immunodeficiency virus-negative patients. J Clin Microbiol 2005, 43:3150-3158

30. Yamori S, Tsukamura M: Comparison of prognosis of pulmonary diseases caused by Mycobacterium avium and by Mycobacterium intracellulare. Chest 1992, 102:89-90.

31. Hanna BA: Diagnosis of tuberculosis by microbiologic techniques. Philadelphia, PA, USA: Little, Brown and Company; 1996.

32. Turenne CY, Tschetter L, Wolfe J, Kabani A: Necessity of Quality-Controlled 16 S rRNA Gene Sequence Databases: Identifying Nontuberculous Mycobacterium Species. J Clin Microbiol 2001, 39:3637-3648.

doi:10.1186/1471-2180-12-137

Cite this article as: Huang et al:: Combined rpoB duplex PCR and $h s p 65$ PCR restriction fragment length polymorphism with capillary electrophoresis as an effective algorithm for identification of Mycobacterial species from clinical isolates. BMC Microbiology 2012 12:137. 